\title{
Technè
}

La science au service de l'histoire de l'art et de la préservation des biens culturels

$40 \mid 2014$

Thérapéia. Polychromie et restauration de la sculpture dans l'Antiquité

\section{Un pied de meuble en forme d'avant-train de lion du département des Antiquités égyptiennes du Louvre : remaniement antique et intervention moderne}

Furniture leg in the shape of the forepart of a lion in the Department of Egyptian Antiquities at the Louvre: antique reworking and modern operation

Noëlle Timbart, Laure de Guiran, Hélène Guichard, Sandrine PagèsCamagna et Juliette Langlois

\section{OpenEdition}

Journals

Édition électronique

URL : https://journals.openedition.org/techne/3461

DOI : 10.4000/techne.3461

ISSN : 2534-5168

Éditeur

C2RMF

Édition imprimée

Date de publication : 26 novembre 2014

Pagination : 53-59

ISBN : 978-2-7118-6218-4

ISSN : 1254-7867

Référence électronique

Noëlle Timbart, Laure de Guiran, Hélène Guichard, Sandrine Pagès-Camagna et Juliette Langlois, « Un pied de meuble en forme d'avant-train de lion du département des Antiquités égyptiennes du Louvre remaniement antique et intervention moderne », Technè [En ligne], 40 | 2014, mis en ligne le 24 juillet 2020, consulté le 09 février 2022. URL : http://journals.openedition.org/techne/3461 ; DOI : https:// doi.org/10.4000/techne.3461

La revue Technè. La science au service de l'histoire de l'art et de la préservation des biens culturels est mise à disposition selon les termes de la Licence Creative Commons Attribution - Pas d'Utilisation Commerciale - Pas de Modification 4.0 International. 
Noëlle Timbart

Laure de Guiran

Hélène Guichard

Sandrine Pagès-Camagna

Juliette Langlois

\section{Un pied de meuble en forme d'avant- train de lion du département des Antiquités égyptiennes du Louvre: remaniement antique et intervention moderne}

Furniture leg in the shape of the forepart of a lion in the Department of Egyptian Antiquities at the Louvre: antique reworking and modern operation
Résumé. Le département des Antiquités égyptiennes du musée du Louvre conserve dans ses collections un élément de mobilier en bois léontomorphe (E 13238). L'étude de la polychromie, des matériaux organiques appliqués en surface, ainsi que l'observation des radiographies menées lors de sa restauration ont permis de répondre à plusieurs questions et notamment de mieux comprendre la stratigraphie complexe de ses couches colorées. Il est apparu que ce pied de meuble a fait l'objet d'une réutilisation, très vraisemblablement durant l'Antiquité. Toutefois, sa nouvelle fonction et son usage demeurent inconnus.

Mots-clés. Protomé, pied de meuble, bois, restauration, polychromie, remaniement antique, vernis, repeints, époque gréco-romaine.
Abstract. A lion-shaped wooden furniture leg (E 13238) is kept found in the collections of the Department of Egyptian Antiquities at the Louvre. The study of its polychromy, the organic materials applied to its surface, and the X-rays taken during its restoration, have enabled us to answer several questions and to better understand the complex stratigraphy of its layers of colour. It became clear that this furniture leg was reused for another purpose, very probably in Antiquity. However, its new function and use remain unknown.

Keywords. Protome, furniture leg, wood, restoration, polychromy, ancient modifications, varnish, repaints, GrecoRoman period.

\section{Introduction}

Au sein de la collection variée de mobilier en bois égyptien, domestique ou funéraire que conserve le département des Antiquités égyptiennes du musée du Louvre, figure un élément isolé dont l'aspect spectaculaire est assuré à la fois par sa nature thériomorphe - la tête, la crinière, le poitrail couvert d'un plastron à damier et les pattes antérieures sont ceux d'un lion - et par sa taille $(60 \mathrm{~cm}$ de haut pour $20 \mathrm{~cm}$ de large et $31 \mathrm{~cm}$ de profondeur). Sculpté en ronde-bosse, polychrome et doré, l'objet a appartenu à un meuble, très vraisemblablement un lit funéraire (fig. 1).

Issu des collections du Cabinet des Médailles de la Bibliothèque nationale, il fut affecté au Louvre en 1907, sans qu'aucune information n'atteste de son histoire matérielle antérieure, si ce n'est la curieuse mention à l'encre sur l'épaule gauche de l'animal: «JULLIOT. Floréal an X.» La date, correspondant aux mois d'avril-mai 1802, et le patronyme indiqué laissent penser que l'objet aurait pu faire partie du fonds du marchand-mercier Philippe-François Julliot (1755-1836), vendu sur saisie à la même époque.
Ce léonin pied de meuble était conservé en réserve depuis son entrée au Louvre et il n'a été que tout récemment remis sur le devant de la scène, grâce au récolement décennal et aux interventions de conservation-restauration qu'il nécessitait. Ces dernières, assorties d'examens et d'analyses, ont permis d'en préciser et d'en enrichir la connaissance, mais aussi de soulever de nouvelles questions.

\section{Description de l'objet}

\section{Le support}

Taillé dans une seule pièce de bois, du figuier sycomore ${ }^{1}$, cet élément de mobilier, dont seules les faces antérieures et gauches sont véritablement sculptées, présente la forme d'un avant-train de lion dont les pattes reposent sur un petit dé aujourd'hui tronqué ${ }^{2}$. Les radiographies (fig. 2) ont mis en évidence la présence de deux mortaises d'assemblages, aujourd'hui comblées par un enduit et recouvertes de repeints les rendant à peine visibles. Celle de la face postérieure, plus

Noëlle Timbart, conservateur du patrimoine chargé des antiquités égyptiennes et orientales, département Restauration, C2RMF (noelle.timbart@culture.gouv.fr). Laure de Guiran, restauratrice de sculptures (ldeguiran@wanadoo.fr).

Hélène Guichard, conservateur en chef du patrimoine, département des Antiquités égyptiennes, musée du Louvre (helene.guichard@louvre.fr) Sandrine Pagès-Camagna, ingénieur de recherche, département Recherche, C2RMF (sandrine.pages@culture.gouv.fr). Juliette Langlois, assistante ingénieur, département Recherche, C2RMF (juliette.langlois@culture.gouv.fr). 


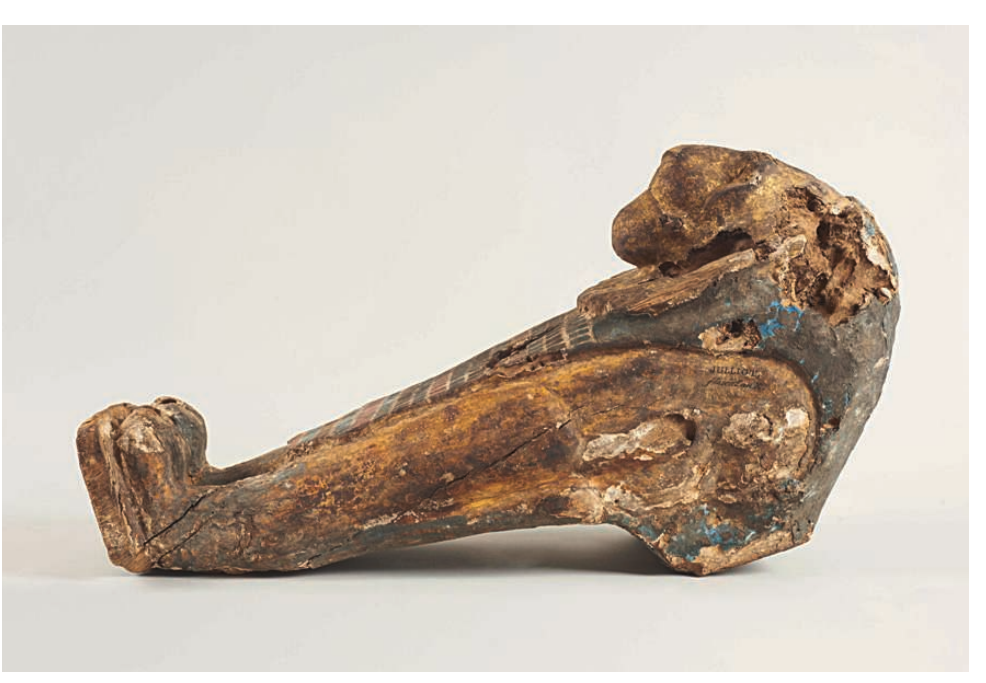

Fig. 1. Protomé de lion après restauration, de face, côté gauche et côté droit, H. 59,7 cm

(E 13238).

(C) C2RMF/Anne

Chauvet.
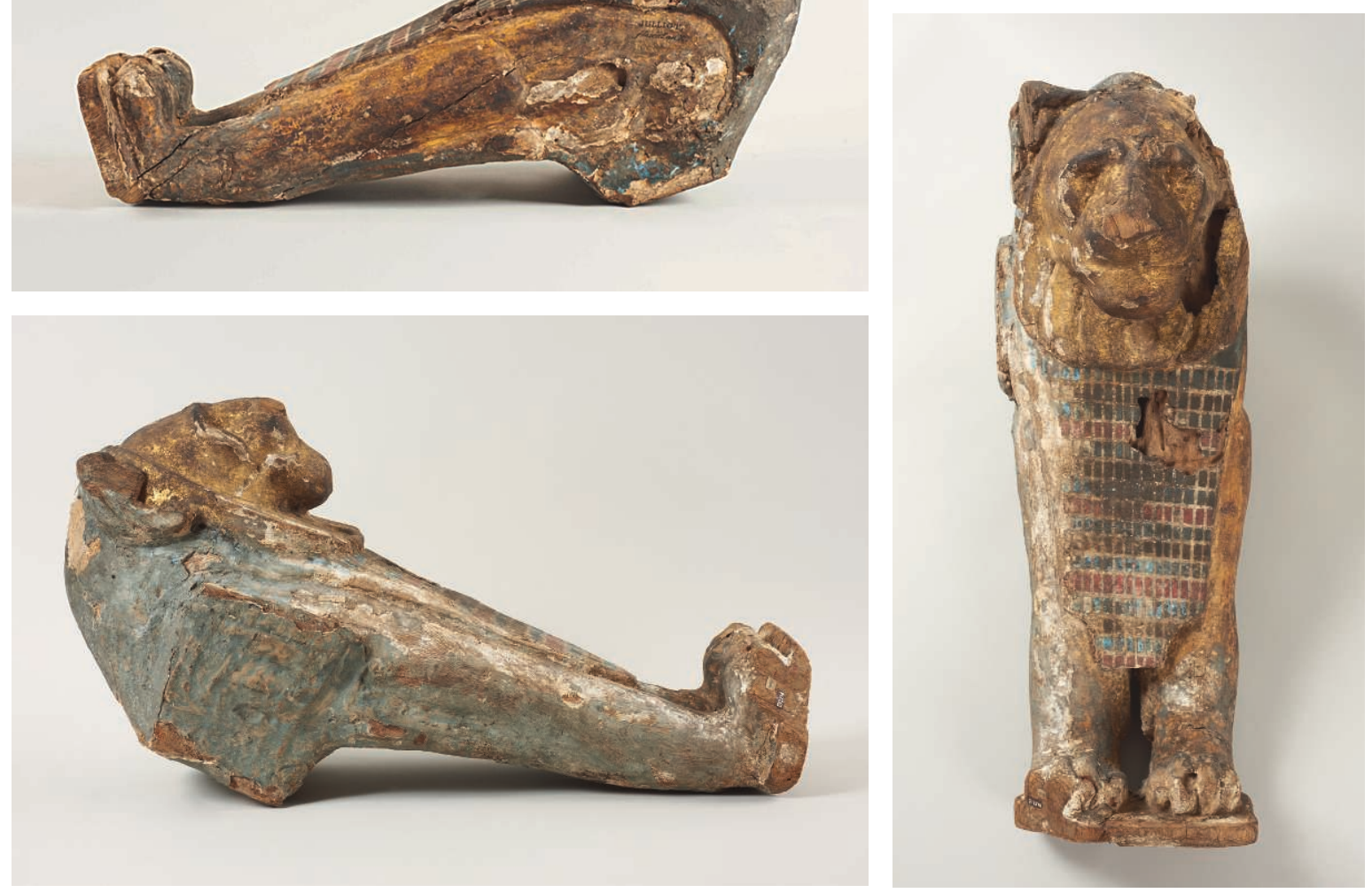

Fig. 2.

Radiographie. (C) C2RMF/Elsa Lambert et Jean Marsac.

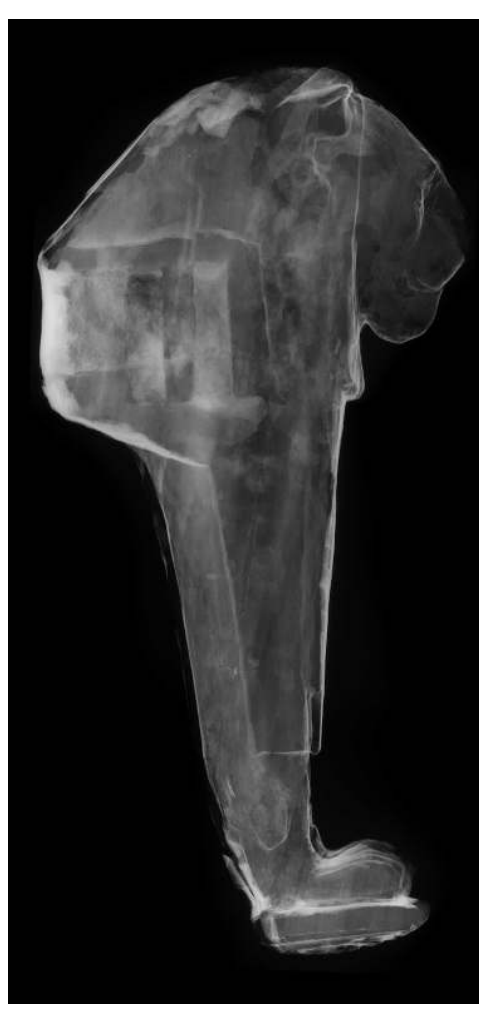

petite, se trouve décentrée vers l'extérieur. À en juger par l'emplacement, en partie supérieure, de ces mortaises destinées à accueillir les tenons de robustes traverses, l'élément constituait très vraisemblablement le pied avant gauche d'un lit funéraire.

Les lits funéraires font partie du mobilier déposé dans les tombes au cours des funérailles. Le cadre du lit, sculpté plus sommairement, faisait référence à la morphologie du lion tandis que le sommier était composé de fibres végétales tressées. En outre, ces lits étaient utilisés au cours de la momification à différentes étapes, le défunt étant déposé sur un lit funéraire en forme de lion stylisé pendant que les prêtres ritualistes et les thanatopracteurs embaumaient le corps. Une fois la momification achevée, la momie pouvait également reposer sur un lit de ce type pour être exposée avant les funérailles, ou bien y être étendue dans la tombe. Les parallèles connus de ce genre d'objet ${ }^{3}$ le rattachent à l'époque gréco-romaine. Ainsi, les fouilles qui ont été menées dans la nécropole de Douch notamment ont mis au jour des momies d'époque ptolémaïque déposées sur un lit léontomorphe identique $^{4}$.

De nombreuses scènes ayant trait à la momification montrent l'emploi de tel mobilier, sur le décor de cercueils, comme par exemple celui de Djedbastetioufankh provenant 


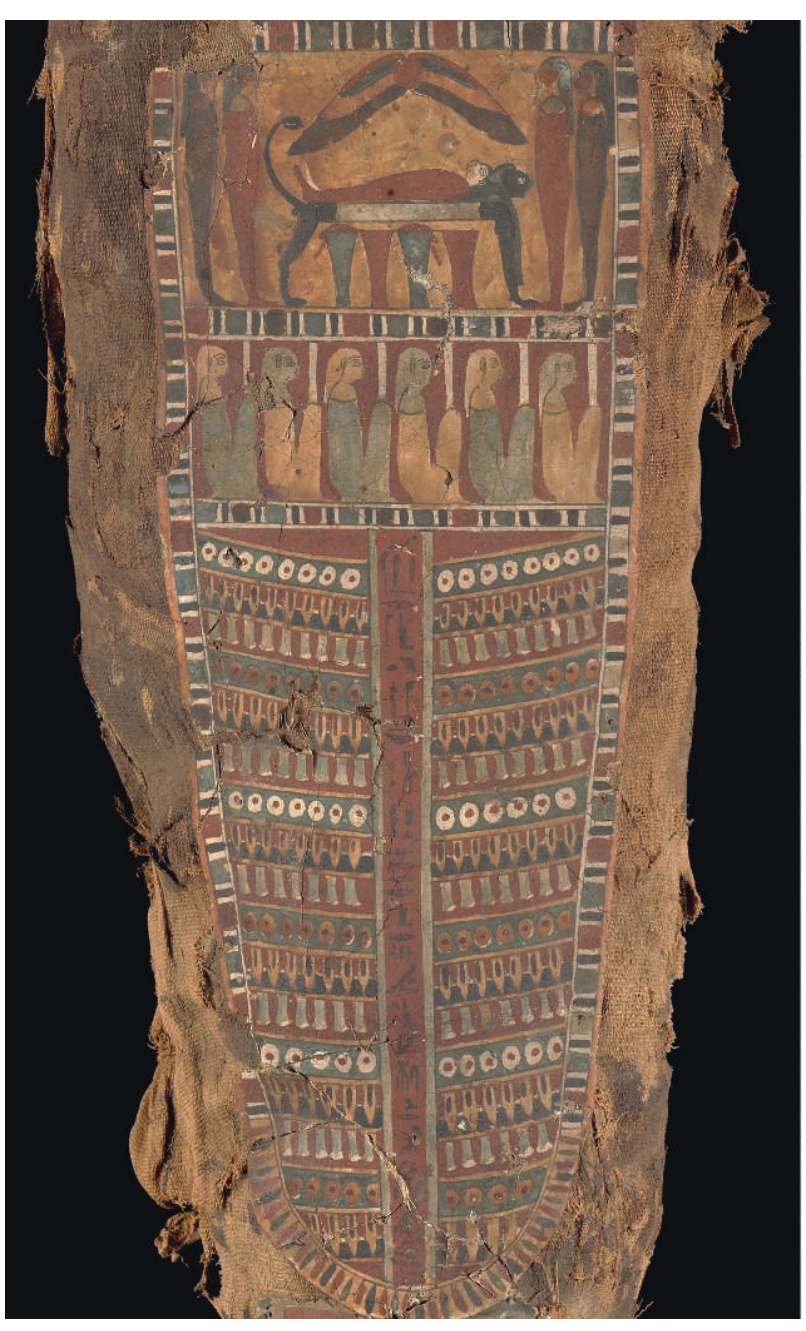

Fig. 3. Représentation d'un lit funéraire léontomorphe sur le cartonnage de la momie d'enfant du Musée d'histoire locale de Rueil-Malmaison, environ 350 av. J.-C. (C) C2RMF/Anne Chauvet. de El-Hibeh et conservé au Pelizaeus Museum d'Hildesheim (II ${ }^{\mathrm{e}}-\mathrm{I}^{\mathrm{er}}$ siècle av. J.-C.), mais aussi sur des cartonnages d'époque ptolémaïque, ou sur les parois de tombes (tombeau de Petoubastis, oasis de Dakhla, $\mathrm{I}^{\text {er }}$ siècle ap. J.-C.) ${ }^{5}$. Dans ces scènes, le dieu Anubis se penche sur le mort dont la tête se trouve systématiquement du côté des têtes de lion, les vases canopes étant figurés sous le lit. D'autres décors montrent le lit funéraire sur lequel la momie repose, encadré par les quatre fils d'Horus (fig. 3).

\section{La couche picturale}

Le décor polychrome est constitué de plusieurs couches. La polychromie originale est appliquée sur une préparation blanche et se compose de la façon suivante: la tête dorée; les pattes jaunes et la coiffe bleue; un plastron à damier bleu, rouge et vert. Diverses strates, mises en évidence par un examen sous loupe binoculaire, recouvrent partiellement cette polychromie originale. La succession de ces strates reste toutefois difficile à interpréter en l'absence d'analyses. Ainsi, le jaune des pattes et la dorure sont recouverts d'un traitement de surface, d'aspect translucide, brillant et épais sur les pattes et plus brun, mat et fin sur le mufle (fig. 4 et 5) qui semble d'origine organique. Une couche de couleur jaune vif visible sous la dorure apparaît également peu habituelle. Un repeint bleu, d'aspect plus terne que l'original et aujourd'hui très assombri, notamment par l'encrassement, a été appliqué sur un matériau blanc (enduit) qui comble les mortaises et

Fig. 5. Couche de traitement de surface translucide et brillante, de type cire d'abeille appliquée sur les pattes. Détail sur la patte gauche de la couche organique jaune.

(C) Laure de Guiran.
Fig. 4. Couche de traitement de surface organique brunie appliquée sur l'or du museau et de la crinière. () Laure de Guiran.

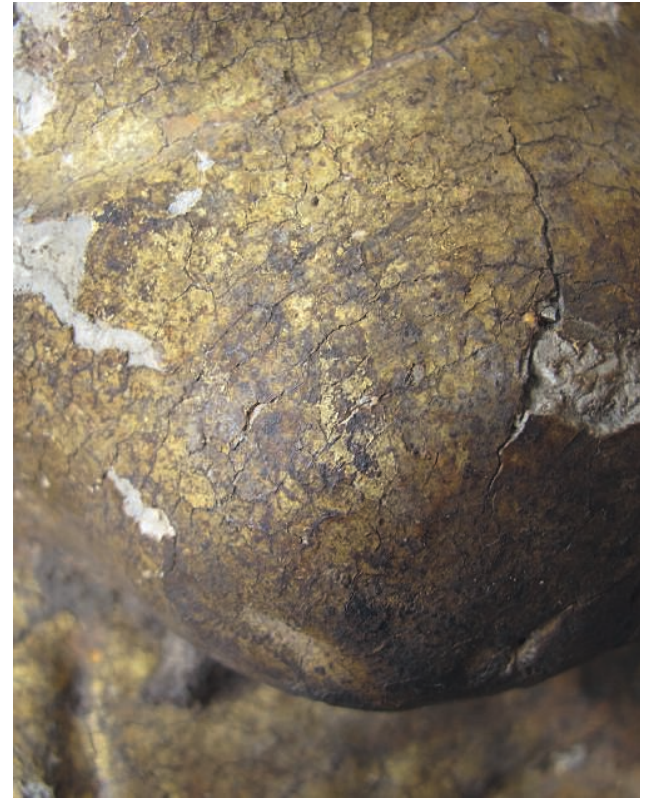

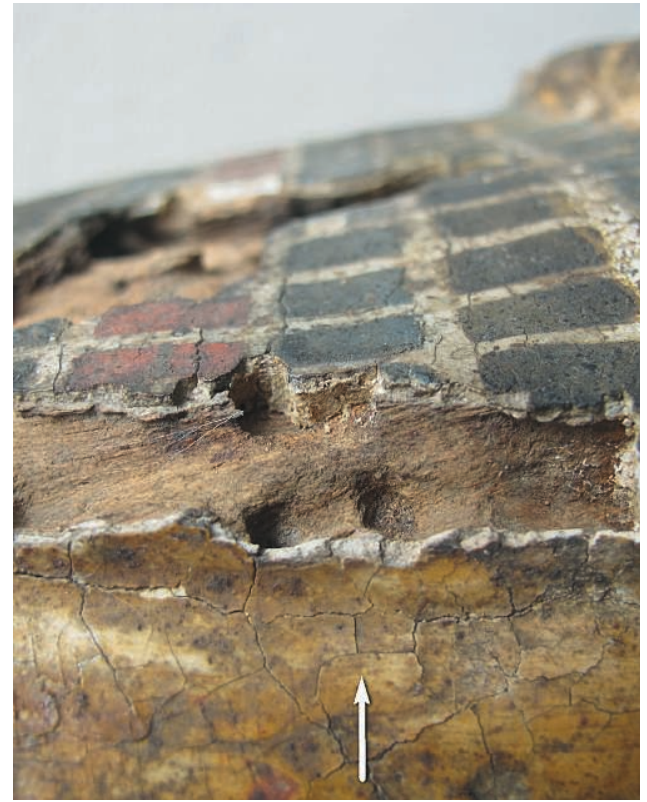




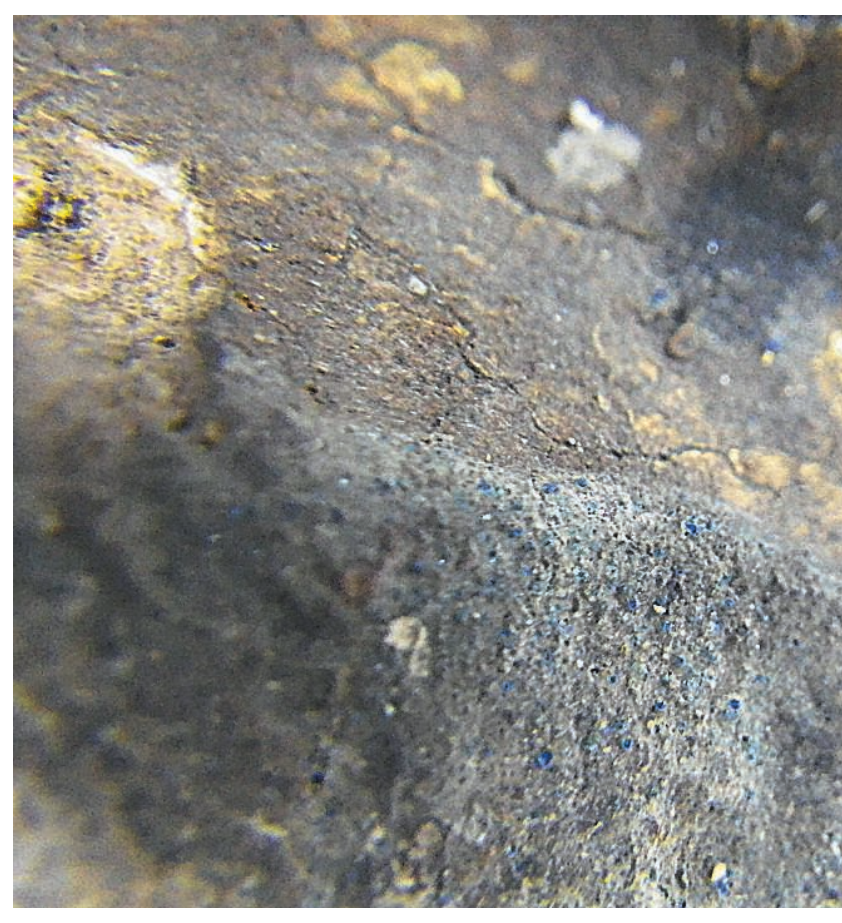

recouvre, ponctuellement, la polychromie originale sur la face postérieure, le côté droit et le dessus de la tête (fig. 6).

\section{Une nécessaire consolidation}

Deux catégories principales d'altération ont été observées sur cet objet: des désordres structurels concernant le support et des altérations de surface.

Le bois comporte les marques d'une ancienne attaque d'insectes xylophages, très vraisemblablement des termites. Les pertes de matière sont importantes et la sculpture est ponctuellement traversée de galeries affleurant en surface. La cohésion du bois est, par endroits, fragilisée par une ancienne attaque de champignon lignivore.

La couche picturale présentait d'importants soulèvements, sous forme de larges cloques, au sommet de la crinière et sur la coiffe, ou de plus légères pertes d'adhérence. Des risques d'enfoncement de la couche picturale étaient également à craindre dans les zones de bois fragilisées. Enfin, la lisibilité de cette œuvre était perturbée par le fort encrassement mais aussi par l'aspect irrégulier du repeint bleu et l'aspect brun du traitement de surface de l'or.

$\mathrm{Au}$ vu de ces constats, une consolidation d'urgence des fragilités du bois et de la couche picturale a été réalisée afin de faciliter la manipulation de l'œuvre et permettre l'examen de la pièce, mais aussi de stopper les processus de dégradation. Les méthodes de consolidation et de refixage ont été sélectionnées au terme d'une série de tests. Le choix du fixatif s'est porté sur une résine vinylique, du Mowital $\mathrm{B} 60 \mathrm{H}^{6}$ en solution dans de l'acétone. Il a été infiltré à $5 \%$ pour consolider le bois et à $10 \%$ pour le refixage des soulèvements de moindre importance. Mélangé à des microbilles de verre, il a servi au comblement et à la consolidation des cloques
Fig. 6. Repeint bleu

terne débordant sur

la dorure sur la

crinière, au-dessus

de la tête.

(C) C2RMF/Sandrine

Pagès-Camagna.

impossibles à réappliquer. Une concentration à $20 \%$ offrait une cohésion et une adhésion suffisante au maintien de la couche picturale et permettait l'infiltration par seringue (fig. 7). La couche picturale a été renforcée, le temps de l'injection du mélange, par l'application de cyclododécane qui, par sa facilité d'application et sa capacité à se sublimer à température ambiante, permet un maintien temporaire adéquat. Les galeries d'insectes affleurant à la surface ont été consolidées lorsque cela a été possible, par des comblements à base de poudre de bois mélangée à du Mowital B60H.

La stabilisation de toutes ces fragilités a permis une manipulation moins risquée, donc l'observation de la couche picturale dans son intégralité et l'affinement des hypothèses stratigraphiques. Ainsi, on constate que ce pied de meuble a été repeint en bleu partiellement, sur la face latérale droite, une grande partie de la coiffe, le revers et sur l'intégralité des deux mortaises préalablement comblées avec un enduit blanc. L'application de ce bleu sur des cassures, notamment au niveau des oreilles, ainsi que des lacunes de la polychromie confirment qu'il constitue une reprise ponctuelle de l'organisation du décor en masquant les assemblages. Les fines couches organiques de traitements de surface visibles sur l'or

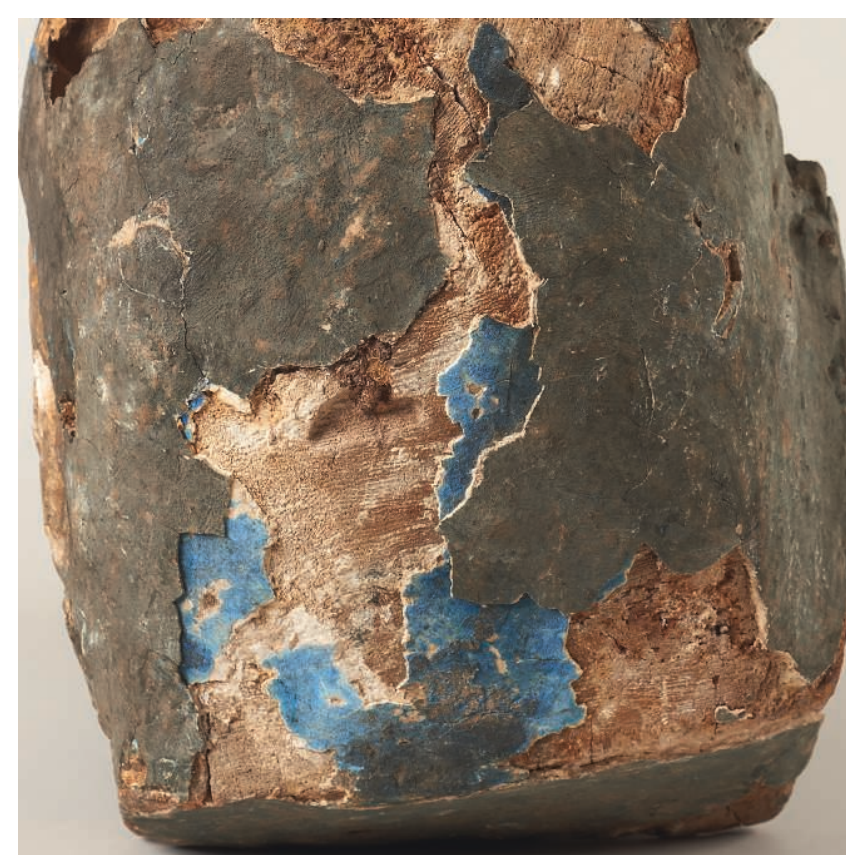

Fig. 7. Soulèvement sous forme de cloque consolidée par infiltration d'un produit de comblement. (C) C2RMF/Anne Chauvet. 
et l'intégralité des pattes restaient quant à elles difficiles à interpréter. Constituaient-elles un traitement de surface original ou des reprises ultérieures ? Et dans ce cas, ces reprises sont-elles contemporaines du repeint bleu?

\section{L'apport des examens et analyses}

Afin de mieux répondre à l'ensemble de ces questions et de comprendre la stratigraphie des couches colorées, un dossier d'imagerie (éclairage naturel et ultraviolet) a été réalisé en amont des analyses et de la restauration. Aucune fluorescence particulière n'apparaît sur les parties comportant un matériau organique.

Des analyses de la polychromie par spectrométrie de fluorescence de rayons $\mathrm{X}$ non invasives, ainsi que la diffraction de rayons X sur micro-prélèvements ont permis de mieux comprendre la nature des interventions et leur succession sur cette pièce mobilière.

Une préparation blanche à base de calcite précède le traitement coloré; un dessin préparatoire noir est visible, indiquant le quadrillage du plastron, et une ligne verticale rouge sépare celui-ci en deux parties égales, répartissant équitablement les carrés du damier (fig. 8).
La crinière est d'un bleu soutenu, de la même teinte que les carrés du plastron.

Le plastron se compose de trois teintes, bleu, rouge et vert: bleu égyptien ${ }^{7}$ avec un peu d'étain, rouge de mercure de type cinabre et vert au cuivre translucide avec un peu d'étain ${ }^{8}$. Les carrés sont mis en couleur, puis du blanc de plomb a été appliqué dans l'intervalle délimité par ces mêmes carrés (fig. 9). Le socle orangé comporte également des pigments arséniés de type réalgar.

Les pattes du lion ont été peintes avec un pigment arsénié, dont la morphologie confirme l'emploi d'orpiment. Le mufle, la crinière et les oreilles ont été dorés à la feuille. Sous la dorure, dans l'oreille droite et également sur le mufle et dans l'œil, des matériaux ocrés apparaissent, renfermant également un peu d'orpiment; il s'agit du support de la feuille métallique.

La nature des pigments employés a évolué au cours de l'histoire égyptienne, et il est ainsi possible d'établir des chronologies d'usage pour certaines teintes, en fonction de la composition des matériaux colorés. La datation de la polychromie de cet objet, au vu de la nature des pigments comme le vert au cuivre organométallique, et surtout en raison de l'emploi du blanc de plomb, ne peut être antérieure à la période ptolémaïque ${ }^{9}$.

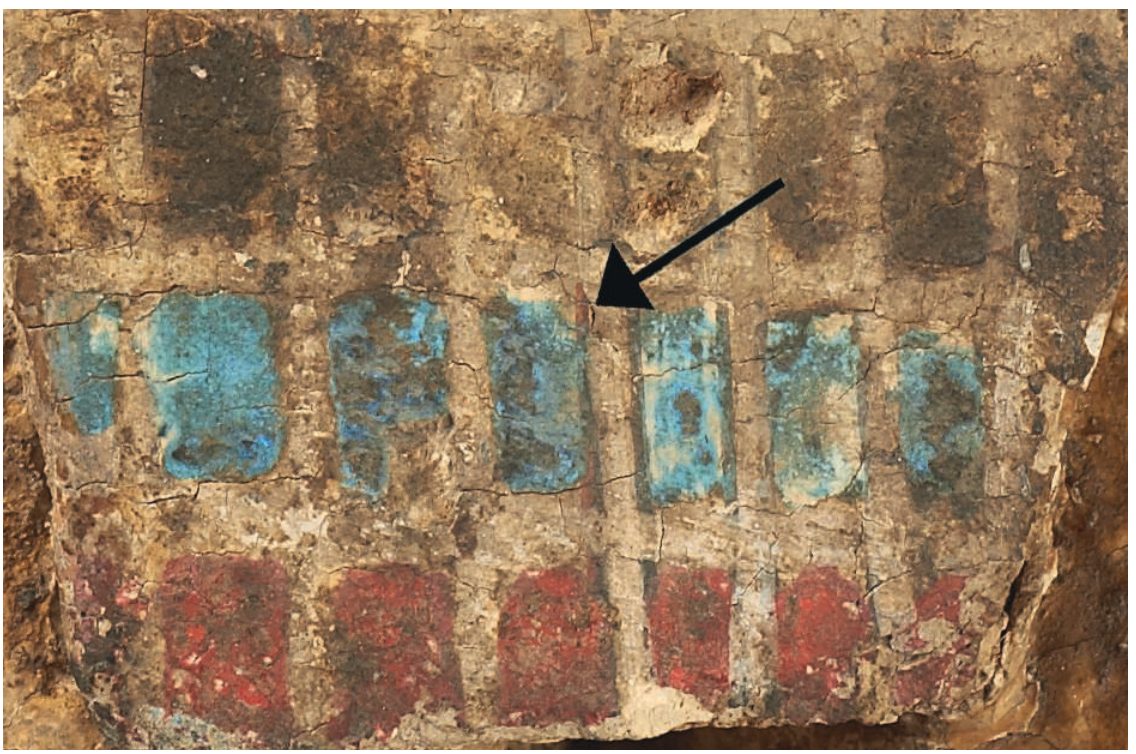

Fig. 8. Dessin préparatoire noir

et partage du plastron en deux parts égales en rouge, signalé par une flèche. (c) $\mathrm{C} 2 \mathrm{RMF} /$ Sandrine PagèsCamagna.

Fig. 9. Contour blanc autour des motifs du plastron. (C) C2RMF/ Sandrine PagèsCamagna.

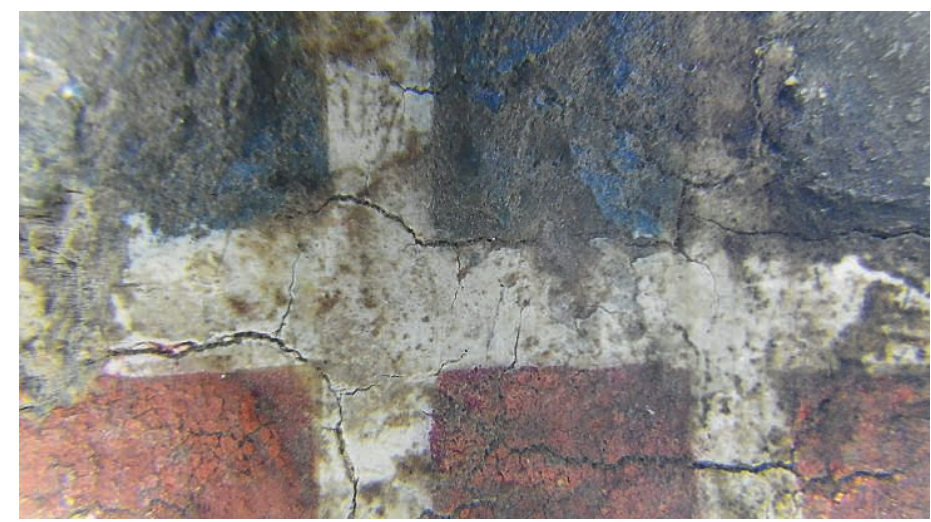




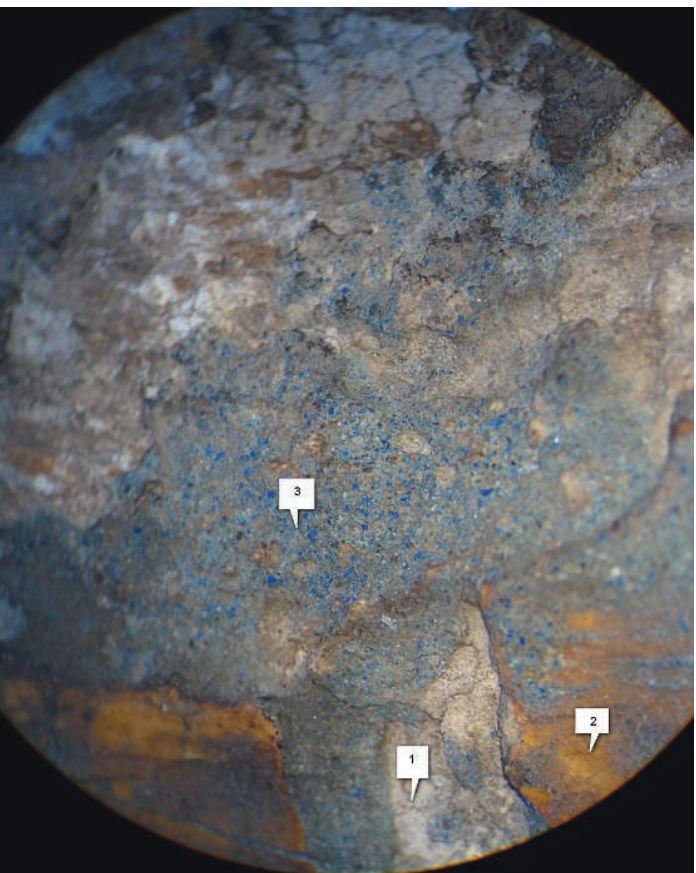

Fig. 10. Repeint bleu visible sur la couche organique.

(c) Laure de Guiran.

Détail stratigraphique sur la patte gauche:

1 , préparation blanche,

2 , couche d'orpiment recouverte

d'une couche organique jaune,

3 , surpeint bleu terne.

L'examen précis de la couche organique couvrant la dorure et les pattes jaunes a permis de constater qu'elle est sous-jacente au repeint bleu (fig. 10). Les profils chromatographiques ${ }^{10}$ des deux échantillons provenant du haut du plastron et de la patte gauche (fig. 11) témoignent de la présence de cire d'abeille légèrement altérée (composés indexés en orange), dont les éléments les plus légers (alcanes et acides gras à longue chaîne hydrogéno-carbonée) ont partiellement disparu, ce qui est fréquent dans le contexte environnemental de conservation égyptien ${ }^{11}$.
Le chromatogramme du micro-prélèvement effectué sur la patte présente également des traces de structures abiétanes oxydées (acide 7-oxodehydroabiétique, acide 15 hydroxydéhydroabiétique et acide 15-hydroxy-7-oxo-déhydroabiétique, indexés en vert) caractéristiques d'une résine de la famille des Pinaceae.

Ces compositions sont compatibles avec les couches de «vernis» d'origine antique égyptienne.

\section{Un remaniement antique ou moderne?}

Cet objet a donc subi plusieurs modifications afin de «réparer » les dommages qu'il a subis, notamment l'attaque des insectes xylophages et les accidents du support (oreille). Les galeries d'insectes ont été bouchées. Les modifications qui ont été apportées à ce pied de meuble l'ont détourné de sa fonction initiale : les mortaises neutralisées, les orifices liés à un assemblage devenant inutiles et le tout a été badigeonné avec un repeint de couleur bleue terne.

Mais à quelle date ces modifications ont-elles été réalisées, dans l'Antiquité ou à une époque moderne?

Le fait d'avoir privé cet objet de toute fonctionnalité a tout d'abord conduit à penser que cette intervention était moderne. En effet, dès la fin du XVIII ${ }^{\mathrm{e}}$ siècle et au début du $\mathrm{XIX}^{\mathrm{e}}$, avec l'attrait suscité par l'Égypte ${ }^{12}$, de nombreux objets égyptiens sont arrivés en Europe et ont circulé. Ils ont pu faire l'objet de remaniements et de restauration, notamment par les marchands. Toutefois, les résultats des analyses ont permis de pencher en faveur d'un remaniement antique.

En effet, le bleu terne qui reprend une partie de la coiffe passe sur le bouchage des mortaises et déborde sur la dorure (fig. 6), sur le front et sur les lacunes de l'oreille droite. Il correspond à un mélange de sulfates de calcium (gypse et bassanite) et de bleu égyptien. Il s'agit du repeint employé lors de la réutilisation de la protomé. Par sa nature spécifique, il remonte à l'époque antique. En outre, l'enduit antique comblant les galeries d'insectes présente la même composition, à base de

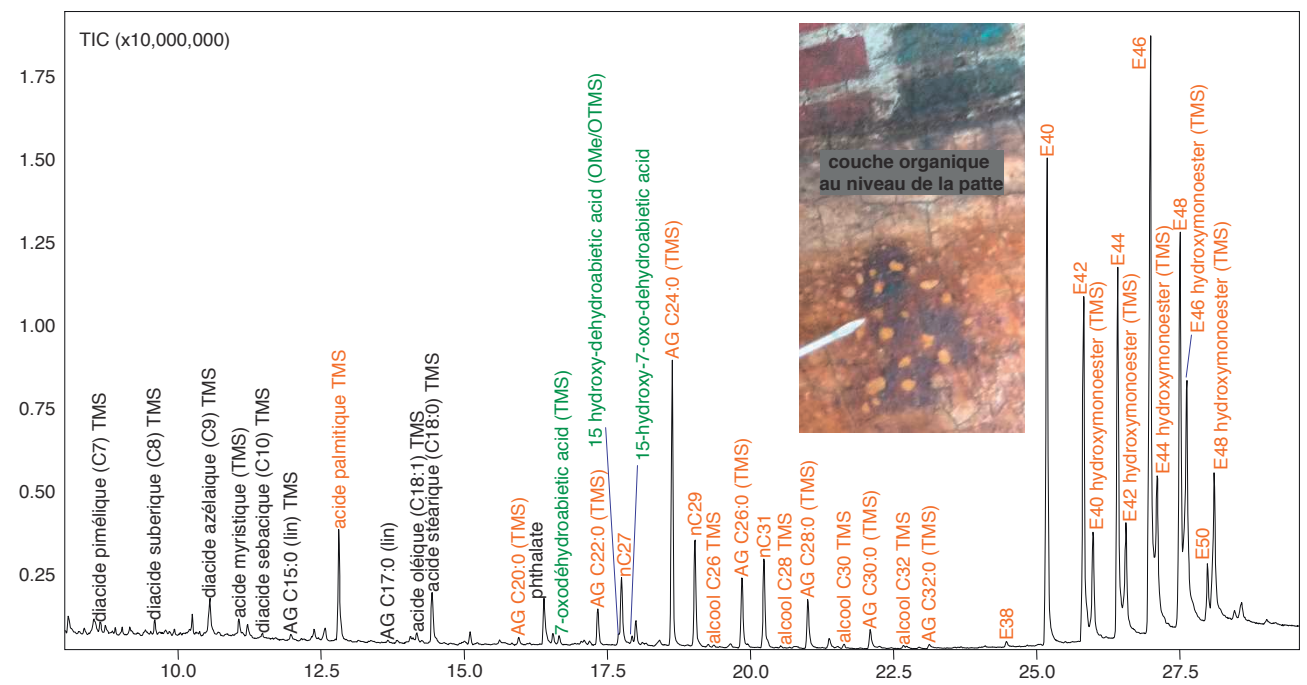

Fig. 11.

Chromatogramme GCMS de la couche organique de la patte gauche. (c) C2RMF/Juliette Langlois. 
sulfates de calcium, que ceux utilisés en bouchage des mortaises et sous le dé ${ }^{13}$. Ces enduits sont bien contemporains du second traitement polychrome. L'association des sulfates de calcium, gypse et bassanite ${ }^{14}$, dans les enduits et dans la couche de bleu terne, ainsi que la nature du pigment bleu, bleu antique de synthèse, sont en faveur d'une intervention antique.

De même, on peut s'interroger sur l'origine de l'objet: est-ce que cet objet provient d'une tombe et aurait été réutilisé dans le monde des vivants, ou bien n'est-il jamais allé jusqu'à la tombe?

Le réemploi était une pratique courante en Égypte, aussi bien pharaonique que tardive ${ }^{15}$, et la fonction initiale des objets - tout ou partie - pouvait être soit conservée, soit détournée pour d'autres usages, ce qui est vraisemblablement le cas de cette protomé. Si de nombreux exemples d'objets réparés par les Égyptiens afin de prolonger leur usage sont parvenus jusqu'à nous, notamment dans le cas du mobilier funéraire, peu d'exemples de modification d'usage sont attestés. Ici, la nouvelle fonction de l'objet est inconnue. Ce dernier ne peut être maintenu en position verticale sans support, mais il est difficile de comprendre comment il était positionné et employé. Il semblerait que son rôle soit devenu purement décoratif, voire cultuel.

\section{Conclusion}

L'opération de conservation-restauration qui a été menée sur cette protomé de lion a permis de stabiliser les fragilités de sa couche picturale tout en recueillant des informations précieuses sur son histoire matérielle. Les analyses et examens ont éclairci sa stratigraphie complexe en répondant aux questions qui se posaient sur la chronologie des applications des couches colorées et de la couche organique. Le niveau d'intervention a pu être choisi correctement: le repeint bleu antique a été conservé sur l'intégralité de l'œuvre et le niveau de nettoyage s'est restreint à un dépoussiérage suivi d'un léger décrassage en prenant garde d'épargner les différentes couches organiques. Néanmoins, à l'issue de ce travail, les motifs qui ont présidé à la transformation de l'objet et la fonction qui a pu, à cette occasion, lui être assignée, n'ont pas été déterminés. Mais le bouchage des mortaises suggère qu'il est devenu objet purement décoratif privé de toute fonctionnalité originelle et se suffisant à lui-même (quoiqu'un système de fixation ait néanmoins été prévu pour le maintenir à la verticale).

\section{Notes}

1. Analyses micrographiques Xylodata, Dr Victoria Asensi Amorós, expert micrographe des bois. Cette essence de bois est peu communément utilisée pour les éléments de mobilier.

2. La plupart des éléments mobiliers égyptiens empruntent leur forme au monde animal et végétal. Très souvent, les pieds des fauteuils, chaises, etc., sont des pattes d'animaux qui reposent sur des petits dés.

3. Dans les collections françaises, voir par exemple le catalogue ${ }^{\circ} 173$ du musée de Picardie à Amiens, Perdu et Rickal, 1994, p. 99.

4. Tombe $\mathrm{n}^{\circ}$ 20, nécropole de Douch, oasis de Kharga, Charron, 2002, p. 93.

5. Dunand et Lichtenberg, 2001, p. 117, 132, 133; Charron, 2002, p. 45, 46 (tombe de Kôm el-Chougafa), p. 97 (cercueil de Djedbastetioufankh), p. 103 (cartonnage d'une momie, tombe E35, nécropole d'El Deir), p. 154 (Livre des morts de Tcha-HapiImou, Basse Époque, musée du Louvre).

6. Composition: polymère de vinylbutiral.

7. Le bleu égyptien est un pigment synthétique formé par traitement thermique dont la couleur provient de l'ajout d'un matériau cuivreux; les restes d'étain signent l'emploi de résidus de bronze alliant cuivre et étain.

8. La nature translucide de la couche ainsi que l'absence de réelle granulométrie permettent d'exclure la nature minérale du pigment, comme la malachite ou le vert égyptien; il s'agit encore d'un pigment de synthèse obtenu à partir de résidus de bronze, mais plutôt d'un organométallique, issu d'un traitement chimique.

9. Scott et al., 2009; Pagès-Camagna et Guichard, 2010.

10. Les deux micro-prélèvements de la couche organique de surface ont été analysés en chromatographie en phase gazeuse couplée à la spectrométrie de masse afin d'identifier la nature de cette substance naturelle.

11. Regert, 2001

12. Fiechter, 2009

13. Les quatre micro-échantillons correspondant aux enduits de bouchage, à la préparation blanche ancienne et au repeint bleu terne ont été analysés par diffraction de rayons X et microspectrométrie Raman. 14. Les préparations des portraits du

Fayoum comportent notamment de la bassanite, avec un peu de gypse.

15. Harrington, N., 2012; Mallinckrodt, C. S., 2013.

\section{Bibliographie}

Charron, A. (dir.), 2002, La mort n'est pas une fin-Pratiques funéraires en Égypte d'Alexandre à Cléopâtre, Musée départemental de l'Arles antique.

Dunand, Fr. et Lichtenberg, R., 2001, Les momies et la mort en Égypte, éditions Errance.

Fiechter, J.-J., 2009, Egyptian Fakes: Masterpieces that Duped the Art World and the Experts Who Uncovered Them, Flammarion.

Harrington, N., 2012, "Reuse of tombs, cemeteries and funerary equipment: prioritising the living over the dead?" dans Living with the Dead: Ancestor Worship and Mortuary Ritual in Ancient Egypt, Oxbow Books.

Langlois, J., octobre 2013, Rapport d'étude, $C 2 R M F, n^{\circ} 26986$.

Mallinckrodt, C. S., 2013, "This Old Foot: Identification of Ancient and Modern Reuse in a Ptolemaic Child Sarcophagus", April 24, 2013 North American Graduate Programs in the Conservation of Cultural Property Conference, Los Angeles.

Pagès-Camagna, S. et Guichard, H., 2010, "Egyptian colours and pigments in the French collections through 30 years: physico-chemical analyses on 300 objects in the laboratory", International symposium on Decorated Surfaces on Ancient Egyptian Objects: Technology, Deterioration and Conservation, 6-9 september 2007, Cambridge, p. 25-32.

Perdu, O. et Rickal, E., 1994, La collection égyptienne du musée de Picardie, Paris, RMN.

Regert, M., Colinart, S., Degrand, L., Decavallas, O., 2001, "Chemical alteration and use of beeswax through time: accelerated ageing tests and analysis of archaeological samples from various environmental contexts", Archaeometry, 43, p. 549-569.

Scott, D. A., Warmlander, S. S., Mazurek, J. et Quirke, S., 2009, "Examination of some pigments, grounds and media from Egyptian cartonnage fragments in the Petrie Museum, University College London", Journal of Archaeological Science, 36, p. 923-932. 